\title{
Deducción de los Estados de Conmutación para los Inversores Multinivel VSC-MMC. Descripción Orientada a la Enseñanza en Ingeniería
}

\author{
Nicolás Muñoz-Galeano, Jesús M. López-Lezama y Fernando Villada-Duque \\ Fac. de Ingeniería, Depto Ing. Eléctrica, Grupo de Manejo Eficiente de la Energía - GIMEL, \\ Universidad de Antioquia, Calle 67 No. 53-108, Oficina 19-437, Medellín, Colombia \\ (e-mail: nicolas.munoz@udea.edu.co, jmaria.lopez @udea.edu.co,fernando.villada@udea.edu.co)
}

Recibido Jun. 2, 2017; Aceptado Ago. 8, 2017; Versión final Sep. 23, 2017, Publicado Feb. 2018

\section{Resumen}

Este artículo presenta el detalle de los estados de conmutación para los inversores multinivel VSC-MMC, explicando el cierre y apertura de cada uno de los semiconductores e incluyendo un análisis del intercambio de energía entre los condensadores y bobinas del sistema. El artículo presenta el detalle del principio de funcionamiento haciendo un análisis riguroso de los estados de conmutación de tal forma que estudiantes y profesionales de ingeniería, mediante la lectura de este artículo, podrán entender con mayor facilidad el principio de funcionamiento del inversor multinivel. El análisis se realiza mediante el uso del control de banda de histéresis que permite observar los cambios de la corriente y tensión en las bobinas del inversor. Este artículo también podrá utilizarse como base para obtener las ecuaciones que rigen el comportamiento de los inversores multinivel y diseñar controladores basados en su modelo matemático.

\section{Deduction of Commutation States for VSC-MMC Multilevel Inverters. Description Oriented to Teaching in Engineering}

\begin{abstract}
This paper presents the detail of the commutation states for VSC-MMC multilevel inverters, explaining the closing and opening of each semiconductor and including energy interchange analysis between inductors and capacitors. The paper presents the detail of the operating principle making a rigorous analysis of the commutation states in such a way that students and engineering professionals through the reading of this paper will be able to understand the operating principle of multilevel inverters. The analysis is done through the use of hysteresis band current control that allows observing changes in the currents and voltages of the inverter inductors. This paper can also be used as a basis to obtain the equations that describe multilevel inductors and to design controllers.
\end{abstract}

Keywords: commutation states; hysteresis band control current; multilevel inverter; energy interchange. 


\section{INTRODUCCIÓN}

Este artículo aborda la explicación de los estados de conmutación y el principio de funcionamiento de los inversores multinivel desde una perspectiva académica. La literatura técnica reporta gran cantidad de publicaciones que principalmente incluyen propuestas para topologías de inversores multinivel, (Rodríguez et al., 2002; Rodríguez et al., 2007) presentan revisiones bibliográficas que incluyen las topologías y estrategias de control más comunes para inversores multinivel. A pesar de que los inversores multinivel son ampliamente estudiados por la comunidad científica, la revisión bibliográfica no reporta algún artículo que detalle el principio de funcionamiento o los estados de conmutación para los inversores multinivel. Por esta razón, estudiantes de ingeniería o ingenieros usualmente tienen dificultades para entender artículos que están relacionados con estos tópicos. Este artículo pretende ser una guía para entender su principio de funcionamiento, brindando herramientas y propiciando el entendimiento de artículos científicos o académicos de mayor complejidad.

El inversor multinivel se presenta como una alternativa para obtener tensiones en AC a partir de tensiones en DC en aplicaciones de alta tensión, los conceptos básicos pueden consultarse en (Rashid, 2004) (Rashid, 2011). En (Rodríguez et al., 2002) se presenta un resumen de las topologías de inversores multinivel que se han venido usando de forma tradicional, dentro de las cuales se encuentran: inversor multinivel de diodo fijador (diode-clamped), inversor multinivel de condensador fijador (capacitor-clamped), inversor multinivel con conexiones de inversores en cascada (cascade multicell with separates $d c$ sources) e inversores multinivel híbridos (Zhao et al., 2010). Sin embargo, recientemente se ha empezado a usar, debido a su versatilidad, la topología VSC-MMC como fuente de corriente controlada para algunas aplicaciones que requieren conexión a la red eléctrica (Voltage Source Converter - Multi-Modular Converter) (Martínez-Rodrigo et al., 2015).

Esta topología se ha venido adoptando en diversas aplicaciones de ingeniería: transmisión en HVDC (Gnanarathna et al., 2011), convertidores Statcom (Du y Liu, 2013; Mohamandi y Tavakoli-Bina, 2011), generación eólica (Zhang et al, 2016), generación fotovoltaica (Ge et al., 2017) y motores de inducción (Harz, 2017). Esto debido a que presenta las siguientes ventajas sobre los inversores multinivel tradicionales: 1) Es una topología modular, por lo que es fácilmente escalable. 2) El almacenamiento de energía se realiza en los condensadores de cada módulo o unidad básica. 3) Cuando se implementa el inversor multinivel con un elevado número de niveles, es posible prescindir de filtros y transformadores. 4) Es posible aumentar la frecuencia de conmutación debido a que los interruptores no están sometidos en la etapa de potencia a diferencias de tensión muy altas. Por tales ventajas, la topología VSC-MMC se analizará en este artículo: sin embargo, el procedimiento de análisis presentado puede usarse de forma similar para otras topologías.

Los autores en (Abildgaard y Molinas, 2012) presentan un modelo matemático para el VSC-MMC. Se resalta que para poder modelar se requieren los estados de conmutación. No obstante, ellos no discriminan los estados de los diodos e interruptores, tampoco explican las condiciones para la operación de cada uno de los semiconductores. La explicación propuesta detalla cada uno de los estados de los semiconductores del inversor y presenta las condiciones para su apertura y cierre cuando se inyecta corriente en el lado de alterna a parte de las cargas de la red eléctrica. Para explicar los estados de conmutación del inversor VSCMMC, se propone el uso del control de banda de histéresis de corriente tomando como base la metodología presentada por (Muñoz et al, 2016) para inversores puente H. Se utiliza el control de banda de histéresis debido a que permite obtener el detalle de los estados de conmutación, utilizando las leyes de Kirchhoff es posible determinar si un semiconductor se activa o no. Además, el control de banda de histéresis es un control simple, fácil de entender y muy intuitivo. Muchas aplicaciones de ingeniería y en particular algunas aplicaciones en electrónica de potencia no utilizan el principio de funcionamiento como base para obtener la estrategia de control; por lo que, no es posible conocer con certeza las ventajas y limitaciones que se tienen. Conocer el detalle de los estados de conmutación permitirá el uso de estrategias de control que parten del modelo del sistema (Mejía et al, 2017). Así las cosas, este artículo puede servir de base para la deducción de las ecuaciones de transferencia y control basado en el modelo matemático para inversores multinivel que permiten el control de la corriente o la tensión en la salida del inversor.

\section{ESTADOS DE CONMUTACIÓN DEL INVERSOR MULTINIVEL}

La Fig. 1 muestra la unidad básica de los inversores multinivel la cual permite la adición de niveles en la onda de tensión en su salida. Esta unidad está compuesta por los interruptores $Q_{1}$ y $Q_{2}$ (se muestran como IGBTs en la Fig. 1), y por los diodos $D_{1}$ y $D_{2}$ y por un condensador $C$. Para facilitar el análisis todos los interruptores se consideran ideales; es decir, no se consideran sus pérdidas o curvas de encendido y apagado. La unidad básica tiene como función aplicar o no, en las terminales a y $b$, dependiendo de lo que se requiera, la tensión del condensador $C\left(V_{D C} M\right)$. $V_{D C}$ es la tensión del Bus $D C$ del inversor y $N$ es el número de niveles por brazo. Los estados de conmutación de la unidad básica están definidos por los sentidos de corriente y las polaridades 
de las tensiones que se establecen en las terminales a y $b$. Para explicar los estados de conmutación se utiliza el convertidor multinivel de la Fig. 2 que incluye dos unidades básicas por brazo, en total cuatro unidades por rama.

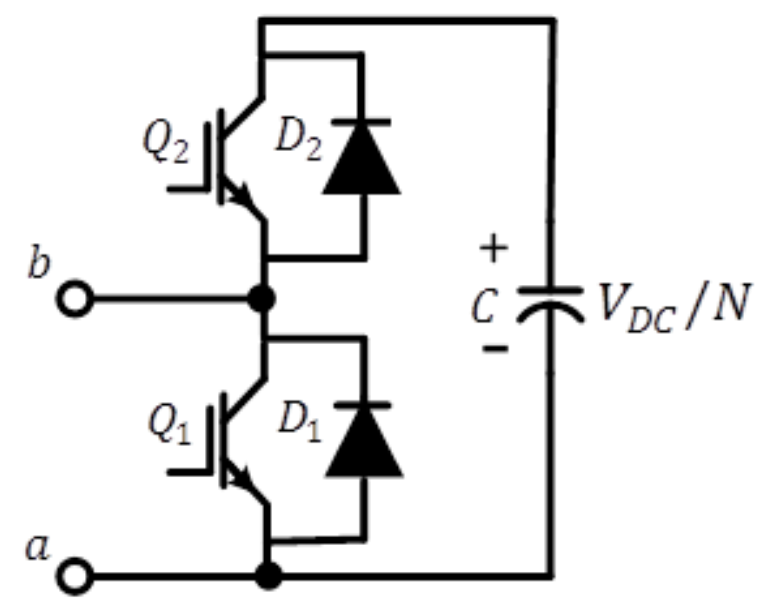

Fig. 1: Unidad básica para el convertidor multinivel VSC-MMC.

El inversor multinivel de la Fig. 2 está compuesto por un Bus DC, un brazo superior y un brazo inferior que se conectan respectivamente a través de los inductores $L_{1}$ y $L_{2}$ a la fuente de tensión alterna $v_{\text {red. }}$ Los brazos inferior y superior componen una de las ramas del inversor (rama $z$ con $z=a, b, c$ ). El Bus DC está compuesto por los condensadores $\left(C_{1}\right.$ y $\left.C_{2}\right)$ con un punto medio $n$ que se conecta al neutro del sistema trifásico. El brazo superior está compuesto por dos unidades básicas y un inductor $L_{1}$ que se conectan entre el Nodo $z$ y el terminal positivo del Bus DC (Bus DC+). El brazo inferior también tiene dos unidades básicas y un inductor $L_{2}$ que se conectan entre el Nodo $z$ y la terminal negativa del Bus DC (Bus DC-). Las referencias para el sentido de la corriente y la polaridad de las bobinas también se ilustran en la Fig. 2, asumiendo que la corriente es positiva cuando la corriente de la red entra al Nodo z. En este artículo no se pretende hacer una contribución al control del dispositivo si no que corresponde a una explicación detallada del principio de funcionamiento del inversor multinivel. Por lo tanto, se considera para el análisis que las tensiones del Bus DC y de las unidades básicas están reguladas a sus valores nominales de operación ( $V_{D C}$ para cada condensador del Bus DC y $V_{D C} 2$ para cada unidad básica).

La Fig. 3 muestra las formas de onda de tensión y corriente en la salida del inversor multinivel. En la parte superior de la figura, la onda de color azul representa la tensión alterna $v_{\text {red }}$ que se conecta en bornes de la salida del inversor y que es impuesta por la red eléctrica. Mientras que en la parte inferior de la figura, la onda de color rojo representa la corriente de referencia $i_{\text {ref }}$ que debe ser seguida por la estrategia de control y por ende debe ser generada por el inversor. La $i_{\text {ref, }}$ que entra al nodo $Z$ de la figura 2 , circula o por la bobina de la rama superior o por la bobina de la rama inferior dependiendo de la polaridad de la tensión de la red: si la polaridad de la red es positiva la corriente circula por la rama superior, mientras que si la polaridad es negativa la corriente circula por la rama inferior.

Se utiliza una corriente $i_{\text {ref }}$ atrasada $90^{\circ}$ con respecto a la tensión $v_{\text {red }}$ para poder contemplar todas las posibilidades de los estados de conmutación, el desfase debe ser impuesto por la estrategia de control. La explicación de los estados de conmutación del inversor se realiza mediante el uso del control de banda de histéresis, no implicando que el control de banda de histéresis deba utilizarse necesariamente para el control del dispositivo. Se utiliza este control para la explicación porque permite establecer con detalle los estados de cada uno de los semiconductores del inversor. Las líneas con guiones grises representan las bandas de histéresis superior (BS) e inferior (BI).

El control de banda de histéresis consiste en que la corriente que inyecta el inversor ( $i_{\text {inv }}$, corriente de color gris con rizado en la figura 3 ) debe seguir la corriente $i_{\text {ref }}$ de tal manera que no se salga de las bandas BS y $\mathrm{BI}$, los estados de conmutación y la secuencia de disparo para que $i_{\text {inv }}$ siga a $i_{\text {ref }}$ se explica en detalle en este artículo. El eje de las ordenadas se divide en cuatro partes de acuerdo a los cinco niveles que se pueden establecer en el inversor multinivel de la Fig. 2. Los niveles de tensión se dividen como sigue: dos niveles de tensión positivos (niveles $1+$ y $2+$ con $V_{1+}=V_{D C} / 2$ y $V_{2+}=V_{D C}$ respectivamente), dos niveles de tensión negativos (niveles 1 - y 2- con $V_{1-}=-V_{D C} / 2$ y $V_{2-}=-V_{D C}$ respectivamente) y un nivel de cero voltios (nivel 0 con $V_{0}$ ). El eje de las abscisas es el eje del tiempo y se divide en 8 zonas de acuerdo a la polaridad de la tensión

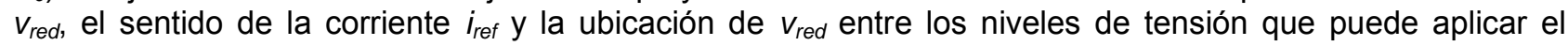
inversor. Las zonas se establecen teniendo en cuenta los niveles de tensión para que la diferencia de tensión en la bobina de salida del inversor no sea superior a la tensión entre niveles. Esto se establece 
como requerimiento en inversores multinivel que usualmente manejan altas tensiones. Las zonas se definen de acuerdo a las condiciones que se presentan en la Tabla 1.

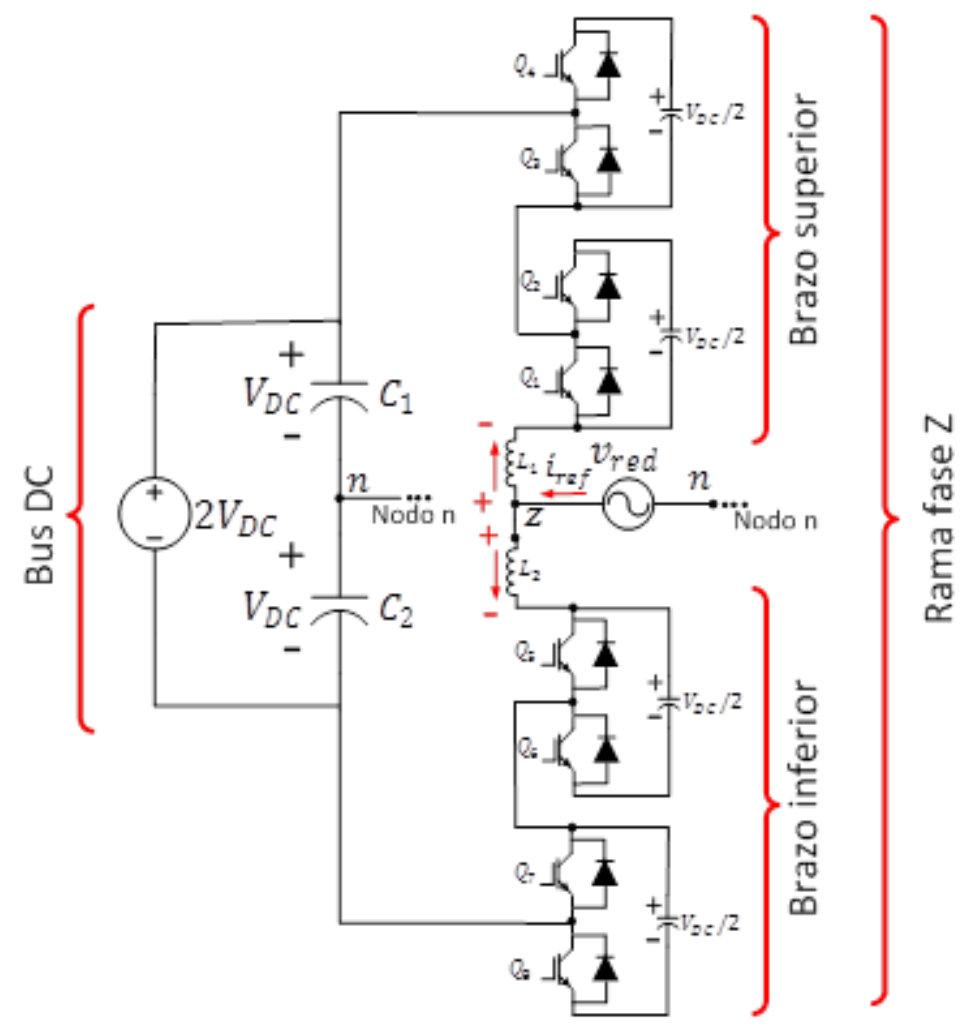

Fig. 2: Representación por fase del convertidor multinivel VSC-MMC de 5 niveles.

Tabla 1: Zonas que establecen los diferentes estados de conmutación del inversor multinivel de la Fig. 2.

\begin{tabular}{|c|c|c|c|c|c|c|c|}
\hline Zona 1 & Zona 2 & Zona 3 & Zona 4 & Zona 5 & Zona 6 & Zona 7 & Zona 8 \\
\hline$v_{r e d}>0$ & $v_{\text {red }}>0$ & $v_{\text {red }}>0$ & $v_{r e d}>0$ & $v_{\text {red }}<0$ & $v_{\text {red }}<0$ & $v_{r e d}<0$ & $v_{\text {red }}<0$ \\
\hline$i_{\text {ref }}<0$ & $i_{r e f}<0$ & $i_{r e f}>0$ & $i_{r e f}>0$ & $i_{r e f}>0$ & $i_{\text {ref }}>0$ & $i_{r e f}<0$ & $i_{r e f}<0$ \\
\hline$V_{0}<V_{r e d}<V_{1+}$ & $V_{1+}<V_{\text {red }}<V_{2+}$ & $V_{1+}<V_{\text {red }}<V_{2+}$ & $V_{0}<V_{\text {red }}<V_{1+}$ & $V_{1}<V_{r e d}<V_{0}$ & $V_{2-}<V_{\text {red }}<V_{1-}$ & $V_{2}<V_{\text {red }}<V_{1}$ & $V_{1}<V_{\text {red }}<V_{0}$ \\
\hline
\end{tabular}

En la parte superior de la Fig. 3 se grafican la tensión de la red y la tensión del inversor; mientras que en la parte inferior se grafican la corriente de referencia, las bandas de histéresis y la corriente del inversor. En cada zona se muestran dos recorridos para la corriente cuyos recorridos se marcaron en sus extremos con puntos rojos y letras. Cada recorrido representa la dinámica de la corriente del inversor de tal modo que es posible establecer la secuencia de conmutación de los semiconductores. Las líneas horizontales negras en la parte superior de la Fig. 3 son las tensiones que el inversor impone mediante la conmutación (modulación) y también se marcan con puntos rojos y letras para poder relacionar cada tramo de la tensión del inversor con su respectivo tramo de corriente.

Para entender el principio de funcionamiento y detallar los estados de conmutación del inversor se debe tener en cuenta lo siguiente: 1) Cuando en bobinas y condensadores la corriente entra por el más (+) el elemento almacena energía, mientras que cuando la corriente entra por el menos (-) el elemento entrega energía. 2) Cuando la corriente aumenta de magnitud independiente de su sentido, la bobina está almacenando energía. Se mencionan dos ejemplos: 2a) En la zona 1, cuando la corriente pasa del punto B al punto $\mathrm{C}$, la corriente se vuelve más negativa pero la bobina está almacenando energía. 2b) En la zona 3 , cuando la corriente pasa del punto $\mathrm{H}$ al punto $\mathrm{I}$, la corriente se vuelve más positiva y también la bobina almacena energía. La bobina cede energía en el caso contrario cuando la corriente disminuye su magnitud, por ejemplo en la zona 1 cuando pasa del punto A al punto B y en la zona 3 cuando pasa del punto $G$ al punto $\mathrm{H}$. 
Los estados de conmutación que permiten realizar todos los recorridos de corriente de la Fig. 3 pueden verse en la Fig. 4 para el semiciclo positivo de $v_{\text {red }}$ y en la Fig. 5 para el semiciclo negativo de $v_{\text {red. }}$ Se explicará a continuación los detalles para obtener los estados de conmutación de la zona 1; de forma similar usando las Fig. 4 y 5 pueden obtenerse el resto.

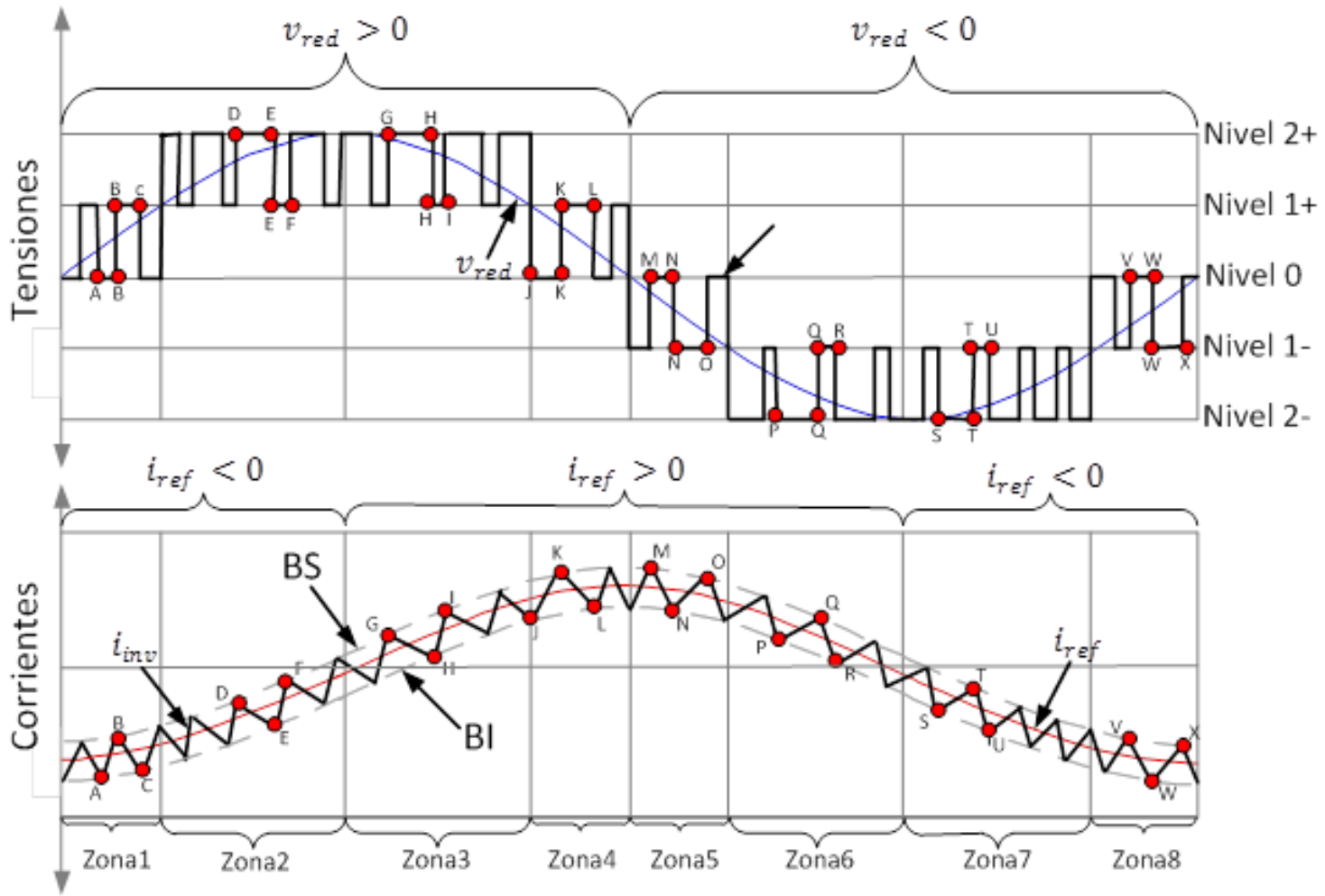

Fig. 3: Formas de onda que permiten la explicación de los estados de conmutación.
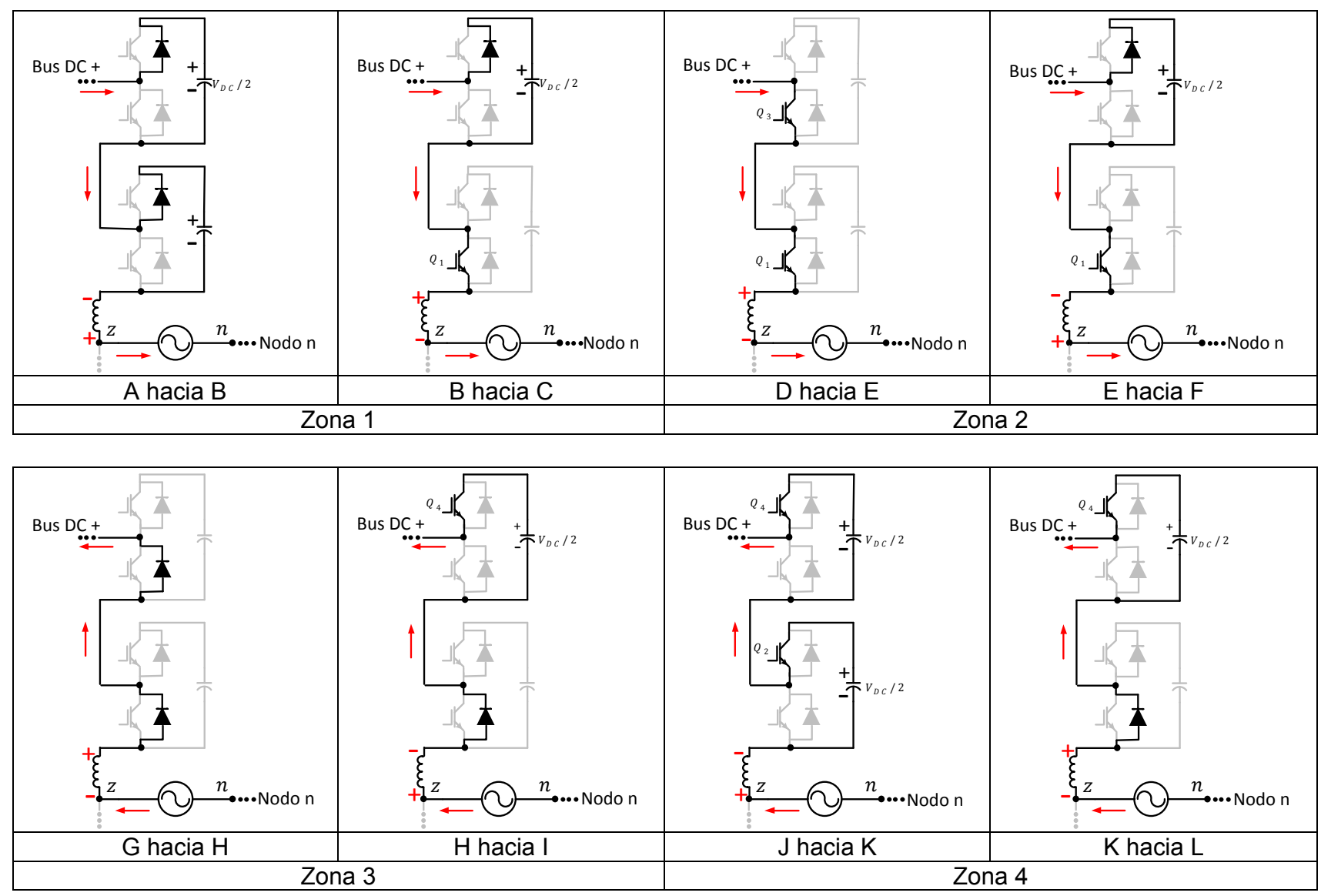

Fig. 4: Estados de conmutación para el semiciclo positivo (rama superior). 
En la zona 1, la tensión $v_{r e d}$ es mayor que cero y se encuentra entre los niveles 0 y $1+$. Por lo que el inversor en sus bornes de salida debe conmutar entre el nivel $0(0 V)$ y el nivel $1+\left(V_{D C} / 2\right)$. También se tiene que la corriente del inversor es menor que cero, debido a que circula de arriba hacia abajo. Para el recorrido desde $A$ hacia $\mathrm{B}$, la corriente del inversor está aumentando de valor volviéndose más positiva, lo que indica que su magnitud está disminuyendo y la bobina está cediendo energía. Esto quiere decir que la polaridad de la tensión en la bobina $L_{1}$ debe imponerse con el menos arriba y el más abajo. Así se garantiza que la corriente del inversor circula desde el menos hacia al más de la bobina de tal forma que cede energía y se descarga.

Para aplicar una tensión que es más negativa en el terminal de arriba de la bobina con respecto al terminal de abajo, la tensión que impone el inversor debe ser menor que la tensión $v_{\text {red; }}$ para que se cumpla esta condición y para que la diferencia de tensión en la bobina no sea superior a $V_{D d} / 2$, el inversor debe aplicar una tensión de $0 \mathrm{~V}$; es decir, el nivel 0 . El nivel 0 puede darse si no se actúa sobre ningún interruptor de tal forma que los diodos superiores de las unidades básicas se polarizan directamente. Esto garantiza que la tensión en la terminal de arriba en la bobina es cero $\left(V_{B U S \_D C+}-V_{B U S \_S U P}-V_{B U S \_I N F}=0\right)$. La polarización de los diodos superiores también garantiza que el sentido de la corriente del inversor es negativa.
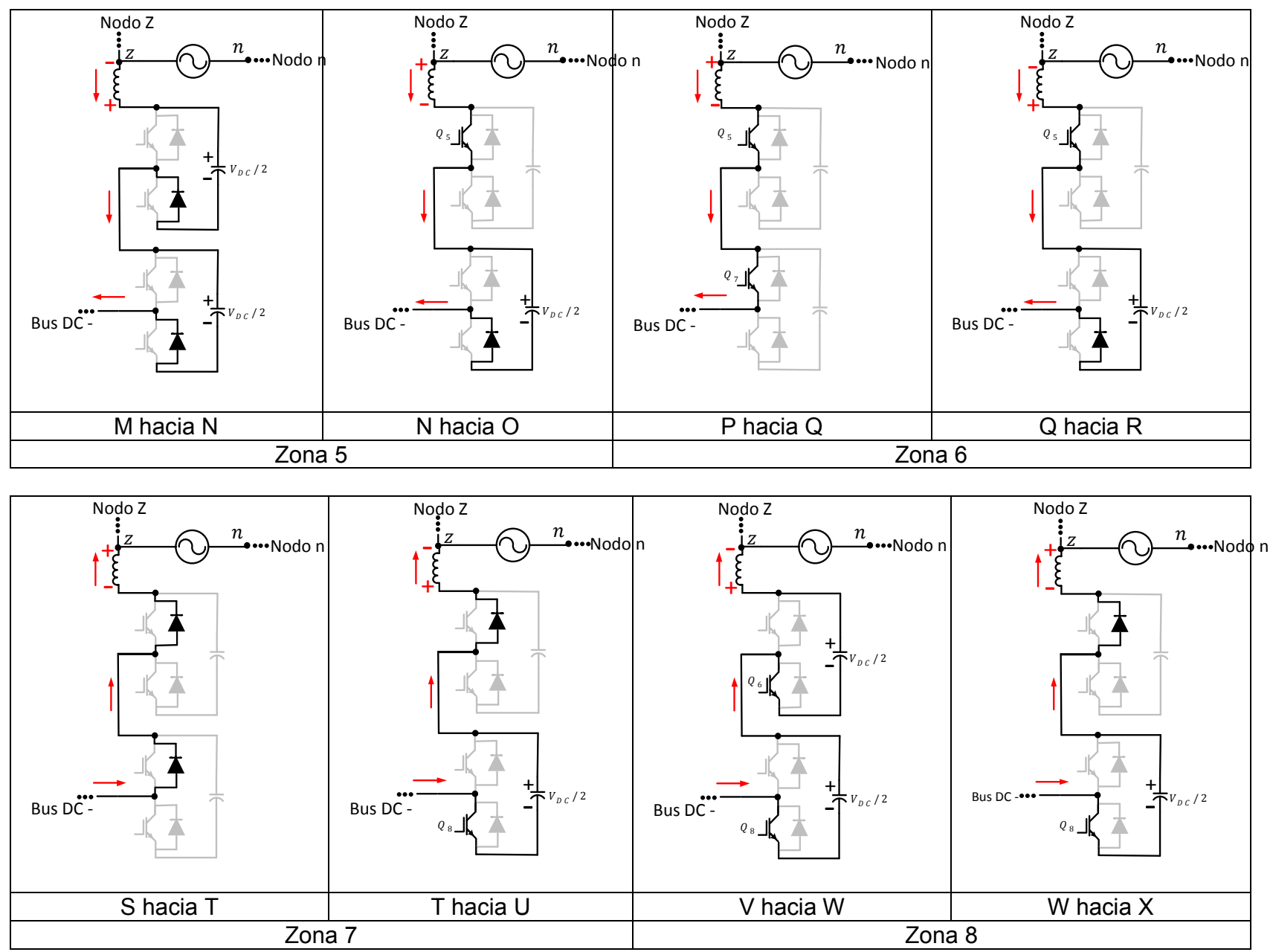

Fig. 5: Estados de conmutación para el semiciclo negativo (rama inferior).

Para el recorrido desde $\mathrm{B}$ hacia $\mathrm{C}$, la corriente del inversor está disminuyendo de valor volviéndose más negativa, lo que indica que su magnitud está aumentando y la bobina está absorbiendo energía. Esto quiere decir que la polaridad de la tensión en la bobina $L_{1}$ debe imponerse con el más arriba y el menos abajo. Así se garantiza que la corriente del inversor circula desde el más hacia al menos de la bobina de tal forma que absorbe energía y se carga.

Para aplicar una tensión que es más positiva en el terminal de arriba de la bobina con respecto al terminal de abajo, la tensión que impone el inversor debe ser mayor que la tensión $v_{\text {redi }}$, para que se cumpla esta condición y para que la diferencia de tensión en la bobina no sea superior a $V_{D C} / 2$, el inversor debe aplicar una tensión de $V_{D C} / 2$, es decir el nivel 1+. El nivel 1+ puede darse si se actúa sobre uno de los dos interruptores inferiores de las unidades básicas. En este artículo se eligió el interruptor inferior de la unidad básica inferior. Cuando este interruptor se cierra evita que la tensión del módulo se reste a la tensión del 


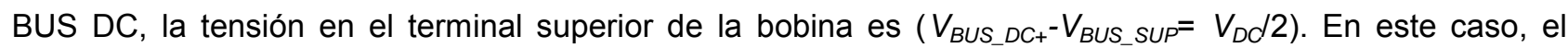
diodo superior de la unidad básica superior se polariza directamente y el interruptor inferior la unidad básica inferior se cierra, garantizando que la corriente del inversor circula desde arriba hacia abajo. La Tabla 2 muestra el resumen de la secuencia de activación para interruptores en cada zona, La letra A indica interruptor abierto y la letra $\mathrm{C}$ indica interruptor cerrado. La lógica de disparo se diseñó para reducir el número de conmutaciones de los interruptores, observe que solo se activa un interruptor a la vez para cambiar de estado de conmutación. Las ecuaciones que permiten el modelamiento del inversor pueden obtenerse fácilmente si se aplican las leyes de Kirchhoff para los estados planteados en las Fig. 5 y 6 , también pueden verificarse usando la Tabla 2.

Tabla 2: Secuencia de activación para los interruptores

\begin{tabular}{|c|c|c|c|c|c|c|c|c|c|c|c|c|c|c|c|c|}
\hline & \multicolumn{2}{|c|}{ Zona 1} & \multicolumn{2}{|c|}{ Zona 2} & \multicolumn{2}{|c|}{ Zona 3} & \multicolumn{2}{|c|}{ Zona 4} & \multicolumn{2}{|c|}{ Zona 5} & \multicolumn{2}{|c|}{ Zona 6} & \multicolumn{2}{|c|}{ Zona 7} & \multicolumn{2}{|c|}{ Zona 8} \\
\hline & $A B$ & $\mathrm{BC}$ & DE & EF & $\mathrm{GH}$ & $\mathrm{HI}$ & JK & $\mathrm{KL}$ & $\mathrm{MN}$ & NO & $P Q$ & QR & ST & TU & VW & WX \\
\hline$Q_{1}$ & $A$ & C & $C$ & C & $A$ & $A$ & $A$ & $A$ & $A$ & $A$ & A & $A$ & $A$ & $A$ & A & $A$ \\
\hline$Q_{2}$ & $A$ & A & $A$ & $A$ & A & $A$ & C & $A$ & A & $A$ & A & $A$ & $A$ & $A$ & $A$ & $A$ \\
\hline$Q_{3}$ & A & $A$ & $C$ & $A$ & A & $A$ & $A$ & $A$ & A & $A$ & $A$ & A & $A$ & $A$ & $A$ & $A$ \\
\hline$Q_{4}$ & $A$ & $A$ & $A$ & $A$ & A & $C$ & C & $C$ & A & $A$ & $A$ & A & A & $A$ & $A$ & $A$ \\
\hline$Q_{5}$ & $A$ & $A$ & $A$ & $A$ & A & $A$ & A & $A$ & A & C & C & C & $A$ & $A$ & $A$ & $A$ \\
\hline$Q_{6}$ & A & A & A & A & A & $A$ & $A$ & $A$ & A & A & $A$ & A & $A$ & A & C & A \\
\hline$Q_{7}$ & A & A & A & A & A & $A$ & $\mathrm{~A}$ & $A$ & A & A & $C$ & A & $\mathrm{A}$ & $A$ & A & $A$ \\
\hline$Q_{8}$ & $A$ & A & $A$ & $A$ & $A$ & $A$ & A & $A$ & A & A & $A$ & A & $A$ & C & C & C \\
\hline
\end{tabular}

En términos generales se puede afirmar que:

1) Cuando no se ejecuta ninguna acción de control, los diodos se polarizan directamente de tal forma que la tensión de la unidad básica se resta a la tensión del Bus DC. Esto permite bajar en un nivel la tensión aplicada en la salida del inversor.

2) Cuando se cierra el interruptor de la unidad básica, el interruptor evita que la tensión de la unidad básica se reste a la tensión del Bus DC (funciona como bypass de la tensión de la unidad básica). En este caso la unidad básica no permite el paso hacia el nivel inferior de la tensión del brazo.

3) De acuerdo al sentido de la corriente del inversor es la polarización de los diodos en las unidades básicas: 3a) Para las zonas 1 y 2 donde las corrientes circulan de arriba hacia abajo (corriente negativa), los diodos superiores de las unidades básicas se polarizan cuando no se ejecuta acción alguna sobre los interruptores. 3b) Similarmente, para las zonas 3 y 4 donde las corrientes circulan de abajo hacia arriba, los diodos inferiores se polarizan directamente. 3c) Un análisis similar puede hacerse para el semiciclo negativo donde los diodos inferiores de las unidades básicas se activan para corrientes negativas, mientras que los diodos superiores se activan cuando la corriente es positiva.

4) El intercambio de energía de las bobinas se realiza cada vez que cambian los estados de conmutación, permitiendo el control de la corriente del inversor.

5) El intercambio de energía con el Bus DC se realiza cada cuarto de ciclo cuando la corriente del inversor cambia de sentido.

6) Se requiere dos bobinas para poder realizar el control de corriente, $L_{1}$ para el brazo superior o semiciclo positivo de $v_{\text {redi }}$; mientras que $L_{2}$ para el brazo inferior o semiciclo negativo de $v_{\text {red }}$. Una sola bobina para ambos brazos ocasionaría conflictos de control ya que $L_{1}$ y $L_{2}$ dependiendo de la secuencia de conmutación deben alternar su polaridad para poder intercambiar correctamente energía con la red eléctrica y los condensadores del bus DC.

\section{PROPUESTA PARA LA ENSEÑANZA EN INGENIERÍA}

La clase de inversores multinivel VSC-MMC debe ser parte del curso de electrónica de potencia para ingenieros electricistas, electrónicos o afines. Los alumnos de electrónica de potencia deben tener conocimientos previos de circuitos eléctricos y de electrónica básica, de tal manera que puedan utilizar las leyes de los circuitos eléctricos y establecer los criterios para la conducción y bloqueo de los 
semiconductores. A continuación se presenta a una propuesta que consiste en un procedimiento detallado para la enseñanza del inversor VSC-MMC en ingeniería.

Todo el conocimiento requerido para cada paso del procedimiento está soportado en este artículo; no obstante, en el caso que se requiera conocimiento adicional se indica la referencia bibliográfica donde se puede consultar. Los objetivos de la clase son: 1) enseñar el principio de funcionamiento, 2) establecer los estados de conmutación para el inversor y 3 ) establecer la secuencia de activación de los interruptores. A continuación se indican los pasos a seguir.

1. Realizar repaso de circuitos eléctricos y electrónica básica. Este paso es opcional, el profesor puede indicar a los alumnos que realicen el repaso en su tiempo de estudio individual.

2. Enseñar el principio de funcionamiento de inversores puente $\mathrm{H}$ que puede consultarse en (Muñoz et al, 2016) para que los alumnos empiecen a familiarizarse con la temática de inversores.

3. Establecer un paralelo entre inversores puente $\mathrm{H}$ e inversores multinivel de tal manera que se establezcan ventajas y desventajas (Rashid, 2004).

4. Mostrar el uso potencial de inversores multinivel en algunas aplicaciones de ingeniería (Gnanarathna et al., 2011; Du y Liu, 2013; Mohamandi y Tavakoli-Bina, 2011; Zhang et al, 2016).

5. Mostrar las topologías de inversores multinivel más comunes, consultar (Rodríguez et al., 2002).

6. Realizar un comparativo entre las topologías de inversor multinivel más comunes, mostrar las ventajas de la topología VSC-MMC sobre las demás (Martínez-Rodrigo et al., 2015). A partir de este paso, la clase debe enfocarse en inversores VSC-MMC.

7. Dibujar o proyectar en la pizarra la unidad básica del inversor multinivel VSC-MMC y definir cada uno de sus componentes, ver Fig. 1.

8. Dibujar o proyectar en la pizarra la topología básica para 5 niveles por fase. No es conveniente emplear más de 5 niveles porque la explicación podría ser muy compleja. Sin embargo, se debe explicar cómo se pueden generar los niveles a partir de la adición de más unidades básicas, ver Fig. 2. En este paso también se deben definir brazos, ramas y fases del sistema.

9. Dibujar o proyectar en la pizarra las formas de onda de: tensión de la red, corriente de referencia, bandas de histéresis, corriente del inversor y tensión del inversor en el nodo Z (ver Fig. 3). Definir correctamente cada forma de onda.

10. Definir las zonas que permiten establecer los diferentes estados de conmutación del inversor. Para esto se debe utilizar la Tabla 1 donde se comparan el voltaje de red y la corriente de referencia. Para poder obtener todas las combinaciones posibles para los estados, se debe definir una corriente de referencia desfasada $90^{\circ}$ con respecto al voltaje de la red.

10. Explicar el comportamiento energético de bobinas y condensadores, definir voltajes y corrientes de referencia.

11. Explicar el control de banda de histéresis que permitirá definir los estados de conmutación (ver Fig. 4 y 5). Indicar que la banda de histéresis se utiliza para la explicación mas no para el control.

12. Tras definir los estados de conmutación, explicar el intercambio energético entre bobinas, condensadores y la red eléctrica usando las leyes circuitales de Kirchhoff.

13. A partir de los estados de conmutación, definir la secuencia de activación de los interruptores. La secuencia de activación debe plantearse de tal manera que se haga el mínimo número de conmutaciones posible (ver Tabla 2).

\section{RESULTADOS DE SIMULACIÓN}

La simulación de la dinámica del inversor para validar la explicación del artículo se realizó en MatlabSimulink (Fig. 6), implementando el esquema el inversor multinivel de la Fig. 2. La tensión del Bus DC se fijó en $340 \mathrm{~V}$, cada condensador del bus DC $\left(C_{1}\right.$ y $\left.C_{2}\right)$ es de $4700 \mu \mathrm{F}$. Cada unidad básica tiene una tensión fija de $85 \mathrm{~V}$ cuyos condensadores son $2300 \mu \mathrm{F}$. Las inductancias de salida $\left(L_{1}\right.$ y $\left.L_{2}\right)$ son $10 \mathrm{mH}$. Los interruptores de las unidades básicas corresponden al bloque "IGBT/Diode" de Matlab-Simulink que permite la implementación de interruptores ideales. La fuente de alterna en la salida del inversor es de $120 \mathrm{Vrms}$. Las resistencias de los condensadores y bobinas se fijaron en $0.001 \Omega$ para evitar singularidades matemáticas en la solución numérica de la simulación.

Los parámetros y valores nominales de la simulación fueron elegidos de acuerdo a la experiencia de los 
investigadores. Se utilizó un bloque S-Function como interfaz de programación y control. El control de corriente de banda de histéresis se fijó con 2 Arms a una frecuencia de $60 \mathrm{~Hz}$ atrasada $90^{\circ}$ con la tensión $\mathrm{AC}$, tal y como se mostró en la Fig. 3. Las bandas de histéresis se calcularon como un porcentaje de la corriente de referencia (3\%). El bloque S-Function tiene como entradas la medida de las corrientes en los brazos superior e inferior y la medida de la tensión de la red; mientras que las salidas son las señales de disparo de cada uno de los interruptores del inversor multinivel. Se programó la S-Function usando los estados de conmutación definidos en las Fig. 4 y 5 . Los resultados de las formas de onda de corriente y tensión del inversor multinivel se muestran en la Fig. 7.
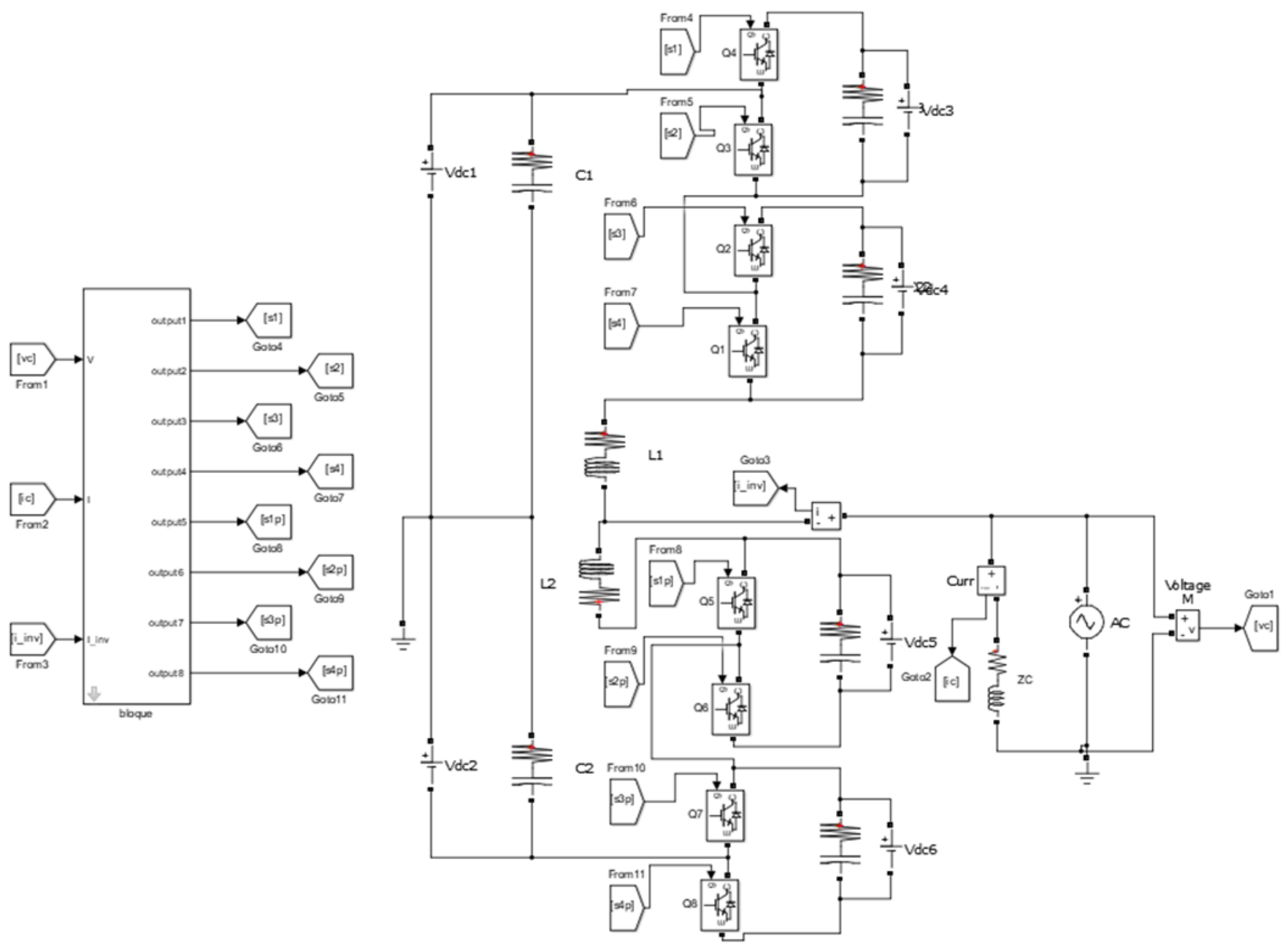

Fig. 6: Simulación implementada en Matlab-Simulink.

La Fig. 7 muestra en su parte superior la tensión de la red, en el medio la corriente del inversor del brazo superior $\left(i_{i n{ }_{-} \text {sup }}\right)$ y en la parte inferior la corriente del brazo inferior $\left(i_{\text {inv_inf }}\right)$. La suma de las corrientes $i_{\text {inv_sup }} \mathrm{y}$ $i_{i n i_{i} \text { inf }}$ corresponden a la corriente de salida del inversor que es senoidal y que está atrasada $90^{\circ}$ con respecto a la tensión de la red. Se remarcan, en las formas de onda de las corrientes $i_{\text {inv_sup }}$ y $i_{\text {inv_inf, las }}$ zonas que delimitan los estados de conmutación tal y como se delimitaron en la Fig. 3. Se puede observar de $i_{\text {inv_sup }} \mathrm{y} i_{\text {inv_inf }}$ que siguen la corriente de referencia senoidal y que los estados de conmutación descritos en este artículo son coherentes. Solo hay que hacer la salvedad que el brazo superior permite el control de las zonas 1, 2, 3 y 4; mientras que el brazo inferior permite el control de las zonas 5, 6, 7 y 8 . Esto implica que hay que fijar una corriente de $0 \mathrm{~A}$ cuando el brazo no se encuentra operativo.

También hay que resaltar que se presentan unos picos de corriente cuando se cambia de zona de operación (ver círculo en la Fig. 6). Esto se da debido a que entre zona y zona cambian los semiconductores que se deben cerrar dadas las condiciones para la apertura y cierre. El control de banda de histéresis no actúa rápido y permite tales picos, un reto para el ingeniero de diseño está orientado a la disminución de estos picos evitando que se salgan de la banda de histéresis o de los objetivos de control. Los picos en este artículo no fueron totalmente corregidos debido a que se trata de un documento orientado a la enseñanza en ingeniería; corregir los picos involucraría un análisis diferente y un objetivo diferente para el artículo. Se puede concluir de los resultados que el principio de funcionamiento descrito y los estados de conmutación 
basado en las condiciones planteadas corresponde al cuerpo de trabajo presentado.
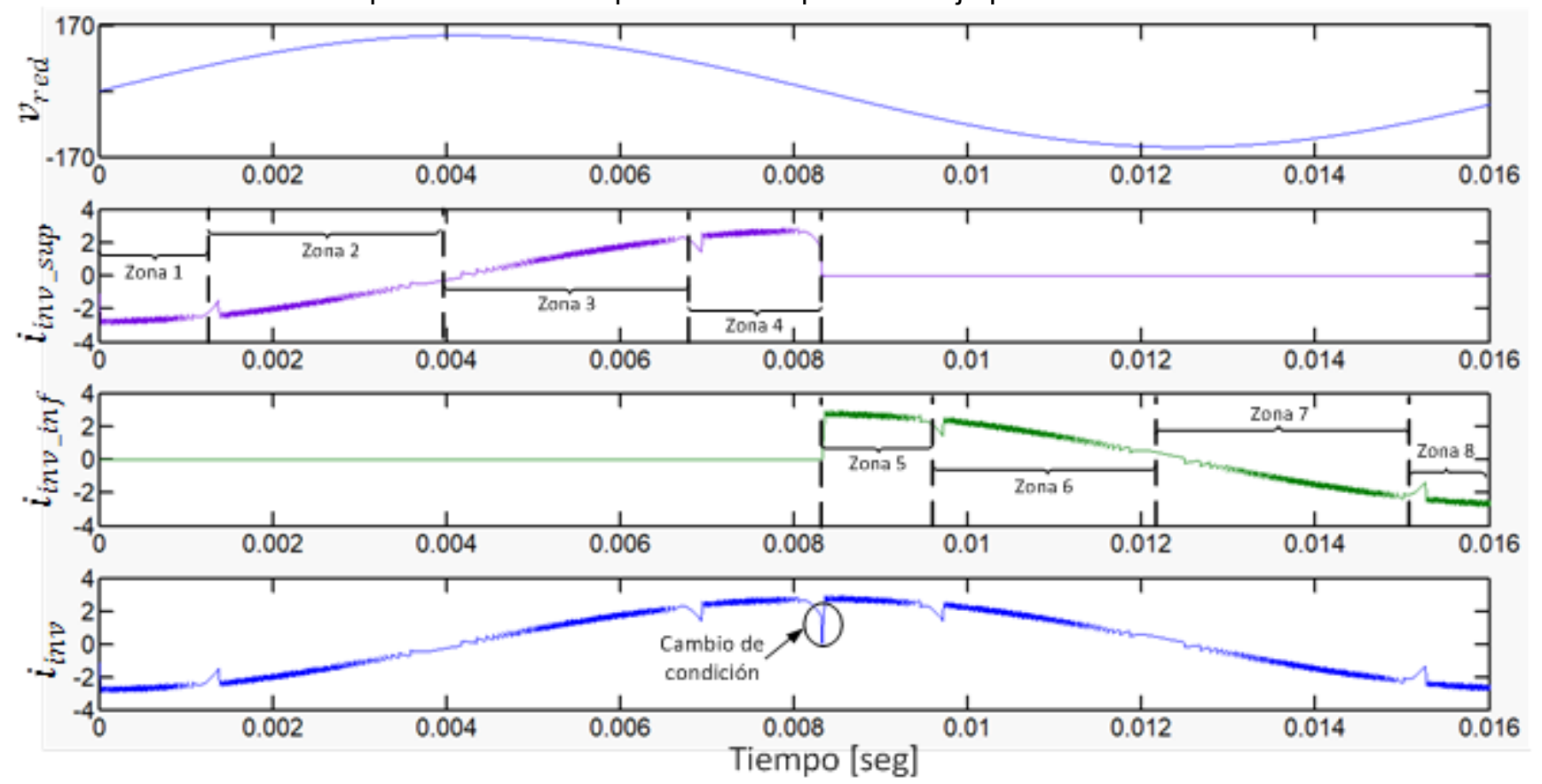

Fig. 7: Resultados de simulación: formas de onda de voltaje y corrientes generadas.

\section{CONCLUSIONES}

El artículo presenta el principio de funcionamiento de inversores multinivel, detallando cada uno de los estados para interruptores y diodos; también se presentan las condiciones que permiten la apertura y el cierre de cada uno de los semiconductores. La explicación de los estados de conmutación se validó mediante resultados de simulación. En cuanto al principio de funcionamiento se puede concluir lo siguiente:

La unidad básica permite disminuir un nivel de la tensión de salida del inversor, para esto no se debe ejecutar acción alguna de control sobre los interruptores del módulo dejando que los diodos conduzcan; por el contrario, si se decide activar el interruptor de la unidad básica, la unidad básica funciona como bypass impidiendo que la tensión del módulo se reste a la tensión del Bus DC y evitando que se pase al nivel inferior de tensión.

La activación de los interruptores y diodos de la unidad básica depende de la tensión de la red, de la corriente del inversor y de la magnitud de la tensión de la red con respecto a los niveles del inversor. Todo esto determina los estados de conmutación del inversor, para lograr un control efectivo se requiere conocer en cada instante el estado de las variables (tensión y corriente) del inversor.

El intercambio de energía de las bobinas se realiza cada vez que cambian los estados de conmutación, mientras que el intercambio de energía con el Bus DC se realiza cada cuarto de ciclo cuando la corriente del inversor cambia de sentido.

Se requieren dos bobinas para poder realizar el control de corriente implicando dos corrientes de referencia. No es posible el uso de una sola bobina ya que se presentan conflictos en el control debido a que la bobina estaría polarizada simultáneamente con tensión positiva y negativa en algunos casos.

\section{AGRADECIMIENTOS}

Los autores agradecen a la Universidad de Antioquia (UdeA) por el apoyo del programa de sostenibilidad y al proyecto CODI $2015-7747$.

\section{REFERENCIAS}

Abildgaard E.N. y M. Molinas; Modelling and Control of the Modular Multilevel Converter (MMC). Elsevier Journal on Energy Procedia, 40(1), 227-236 (2012) 
Du. S. y J. Liu; A study on dc voltage control for chopper-cell-based modular multilevel converters in DSTATCOM application. IEEE Transaction on Power Delivery, 28(4), 2030-2038 (2013)

Ge, B., Y. Liu, H. Abu-Rub y F.Z. Peng; State-of-Charge Balancing Control for Battery Energy Stored QuasiZ Source Cascaded Multilevel Inverter Based Photovoltaic Power System. IEEE Transactions on Industrial Electronics, pp. (99), on-press (2017)

Gnanarathna, U.N., A.M. Gole y R.P. Jayasinghe; Efficient Modeling of Modular Multilevel HVDC Converters (MMC) on Electromagnetic Transient Simulation Programs. IEEE Transaction on Power Delivery, 26(1), 316324 (2011)

Harz, H.; Efficient induction motor drive with multilevel inverter and variable rotor flux. Industrial Electronics (ISIE), 2017 IEEE $26^{\text {th }}$ International Symposium on, Junio 2017, Edinburgh, UK (2017)

Martinez-Rodrigo. F., S. de Pablo y L.C. Herrero-de Lucas; Current control of a modular multilevel converter for HVDC applications. Elsevier International Journal on Renewable Energy, 83(1), 318-331 (2015)

Mejia-Ruiz G. E., N. Muñoz-Galeano y J.M. López-Lezama; Modeling and development of a bridgeless PFC Boost rectifier. Revista Facultad de Ingeniería, Universidad de Antioquia, 82(1), 9-21 (2017)

Mohammadi, H. y M. Tavakoli-Bina; A transformerless medium-voltage STATCOM topology based on extended modular multilevel converters. IEEE Transactions on Power Electronics, 26(5), 1534-1545 (2011)

Muñoz-Galeano, N., J.B. Cano-Quintero y J.M. López-Lezama; Enseñando el Funcionamiento de los Inversores Puente $\mathrm{H}$ : Análisis del Intercambio de Potencia entre Bobinas y Condensadores. Revista Formación Universitaria, 9(1), 117-124 (2016)

Rashid, M.H., Electrónica de potencia: circuitos, dispositivos y aplicaciones, $3^{\mathrm{a}}$ Ed., 1-904. Pearson Educación, México, Naucalpan de Juárez (2004)

Rashid, M.H., Power Electronics Handbook, 3ª Ed., 1-1362. Elsevier, Burlington, USA (2011)

Rodríguez, J., J. S. Lai y F.Z. Peng; Multilevel Inverters: A Survey of Topologies, Controls, and Applications. IEEE Transaction on Industrial Electronics, 49(4), 724-738 (2002)

Rodríguez, J., S. Bernet, B. Wun, J.O. Pont y S. Kouro; Multilevel Voltage-Source-Converter Topologies for Industrial Medium-Voltage Drives. IEEE Transaction on Industrial Electronics, 54(6), 2930-2945 (2007)

Zhang, H., F. Gruson y D. Florez; Christophe Saudemont. Design and Control of a DC Series Offshore Wind Farm Based on HVDC-MMC. Symposium de Genie Electrique, Jun 2016, Grenoble, France (2016)

Zhao, J., X. He, Y. Han, Y. Deng y R. Zhao; Analysis and testing on the switching states transitions of hybrid clamped multilevel inverters. $5^{\text {th }}$ IET International Conference on Power Electronics, Machines and Drives (PEMD 2010), Abril 2010, Brighton, UK (2010) 
